\title{
Combined treatment with extracorporeal shockwaves therapy and an herbal formulation for activation of penile progenitor cells and antioxidant activity in diabetic erectile dysfunction
}

\author{
Seung Hwan Jeon ${ }^{1,2 \#}$, Woong Jin Bae ${ }^{1,2 \#}$, Guan Qun Zhu ${ }^{1,2}$, Wenjie Tian ${ }^{1,2}$, Eun Bi Kwon ${ }^{1,2}$, Ga Eun Kim ${ }^{1,2}$, \\ Sung Yeoun Hwang ${ }^{3}$, Kyu Won Lee ${ }^{1,2}$, Hyuk Jin Cho ${ }^{1}$, U-Syn $\mathrm{Ha}^{1}$, Sung-Hoo Hong ${ }^{1}$, Ji Youl Lee ${ }^{1}$, \\ Sae Woong Kim $^{1,2}$ \\ ${ }^{1}$ Department of Urology, College of Medicine, ${ }^{2}$ Catholic Integrative Medicine Research Institute, The Catholic University of Korea, Seoul, Republic \\ of Korea; ${ }^{3}$ KEMIMEDI, Seoul, Korea \\ Contributions: (I) Conception and design: SH Jeon, WJ Bae; (II) Administrative support: WJ Bae; (III) Provision of study materials or patients: All \\ authors; (IV) Collection and assembly of data: SH Jeon, WJ Bae, SW Kim; (V) Data analysis and interpretation: SH Jeon, WJ Bae, SW Kim; (VI) \\ Manuscript writing: All authors; (VII) Final approval of manuscript: All authors. \\ \#These authors contributed equally to this study and should be considered co-first authors. \\ Correspondence to: Sae Woong Kim, MD, PhD. Department of Urology, Seoul St. Mary's Hospital, College of Medicine, The Catholic University of \\ Korea, 222, Banpo-daero, Seocho-gu, Seoul, 06591, Republic of Korea. Email: ksw1227@catholic.ac.kr.
}

Background: A Korean herbal formulation named KH-204 was reported to have an antioxidant effect in our previous study. We hypothesized that Low-intensity extracorporeal shockwave therapy (Li-ESWT) combined with KH-204 would accelerate the treatment of erectile dysfunction (ED) by enhancing antioxidant. We investigated the synergistic effect of Li-ESWT and KH-204 for ED and explored the mechanism.

Methods: Human umbilical vein endothelial cells (HUVEC) were treated with KH-204 and LI-ESWT in vitro. Fifty 5-week-old male Sprague Dawley rats received an intraperitoneal injection of 5-ethynyl20-deoxyuridine (EdU) which can label live cells, and were randomly divided into five groups: (I) normal; (II) diabetes mellitus-associated erectile dysfunction (DMED); (III) DMED + KH-204; (IV) DMED + LiESWT; and (V) DMED + KH-204/Li-ESWT. Li-ESWT treatment was repeated three times a week every other day for four weeks in group 4 and 5. Meanwhile, rats in group 3 and 5 were orally fed $400 \mathrm{mg} / \mathrm{kg}$ of KH-204 daily for 1 month. Following a 1-week washout period, penile tissues were evaluated by immunostaining and Western blotting.

Results: KH-204 combined with Li-ESWT improved intracavernosal pressure (ICP) in DMED rats. Li-ESWT/KH-204 stimulated HUVEC tube formation and promoted proliferation. Li-ESWT drove progenitor cells to migrate to penile tissue and $\mathrm{KH}-204$ protected penile progenitor cells in the corpus cavernosum. Oxidative stress was relieved by KH-204/Li-ESWT. Treatment with KH-204/Li-ESWT protected penile progenitor cells, which were recruited to the corpus cavernosum by Li-ESWT, from apoptosis via its antioxidant activity. KH-204/Li-ESWT protected penile tissue from oxidative stress by improving the expression of nuclear factor erythroid 2-related factor 2 (Nrf2)/heme oxygenase-1 (HO-1), increasing superoxide dismutase (SOD), decreasing 8-hydroxy-20-deoxyguanosine (8-OHdG), and reducing apoptosis. KH-204/Li-ESWT promoted stromal derived factor-1 (SDF-1) and platelet endothelial cell adhesion molecule-1 (PECAM-1) in DMED rats.

Conclusions: KH-204 protected penile progenitor cells, which were recruited to the corpus cavernosum by Li-ESWT, from apoptosis via its antioxidant activity. The combination of Li-ESWT and KH-204 as a synergy therapy could be a potential and effective treatment for DMED.

Keywords: Low-intensity extracorporeal shockwave therapy; diabetes mellitus; erectile dysfunction (ED); herbal formulation; antioxidant; progenitor cells 
Submitted Oct 01, 2019. Accepted for publication Jan 06, 2020.

doi: $10.21037 /$ tau.2020.01.23

View this article at: http://dx.doi.org/10.21037/tau.2020.01.23

\section{Introduction}

The prevalence of erectile dysfunction (ED), related to obesity and diabetes has increased continuously for decades $(1,2)$. The etiology of diabetes mellitus ED (DMED) is the distal vascular injury caused by hyperglycemia (3). Presently, various therapeutic strategies exist to improve erectile function (4-7). However, for DMED, first-line drug treatments like PDE5i have been associated with poor responses (8). Therefore, there is an urgent need for effective approaches to DMED.

In a very recent meta-analysis (5), an herbal treatment was recognized as an alternative therapy for ED. In 2004, Park et al. (9) found that Ojayeonjonghwan, an herbal formulation known as KH-204, improved ED in aged and diabetic rats. From our extensive research with $\mathrm{KH}-$ 204, we knew it could boost cells' antioxidant capabilities in vivo (10). For DMED patients, increased oxidative stress could contribute to vascular endothelial injury in the corpus cavernosum and subsequently, aggravate ED (11). According to the above studies, $\mathrm{KH}-204$ might be a potential way to improve DMED.

Low-intensity extracorporeal shockwave therapy (LiESWT) has been used to treat musculoskeletal disorders (12), myocardial infarction (13), and even ED (4) for many years, since therapeutic ultrasound has been shown to improve angiogenesis by inducing VEGF expression (14). After Vardi et al. (6) treated ED patients with Li-ESWT clinically, numerous basic studies have explored the mechanism of Li-ESWT $(15,16)$ for ED. Besides inducing angiogenesis, Li-ESWT was also found to promote stem cell homing to the corpus cavernosum in treated ED rats (7). The homing stem cells gathered around the injured tissues and showed regenerative effects on the nerve injuries caused by ED. But under a high oxidative stress environment, further apoptosis of stem cells was inevitable, which diminished the stem cells' effect (17).

In this study, we established a DMED rat model, then administered ESWT or KH-204. And cell experiment was performed for exploring the mechanism of KK-204. We hypothesized that Li-ESWT combined with KH-204 would accelerate the treatment of ED and explored the mechanism of this treatment.

\section{Methods}

\section{Cell culture and experimental design}

Human umbilical vein endothelial cells (HUVECs, Korean Cell Line Bank, Seoul, Republic of Korea) cultured in low glucose-containing Dulbecco's modified Eagle's medium (DMEM) (Gibco, Waltham, MA, US) supplemented with $20 \%$ fetal bovine serum (FBS), (Gibco, Waltham, MA, US) and $5 \mathrm{ng} / \mathrm{mL}$ basic fibroblast growth factor (bFGF) (Cell Signaling Technology, Danvers, MA, US) at $37{ }^{\circ} \mathrm{C}$ and $5 \%$ $\mathrm{CO}_{2}$. After 2 days, the non-adherent cells were removed and the culture media were replaced. The culture media were changed every other day. Cells were passaged when they reached approximately $90 \%$ confluence. HUVECs were divided into four groups: the sham, KH-204, Li-ESWT, and KH-204/Li-ESWT groups. Cells in the KH-204 group were treated with $\mathrm{KH}-204$. Cells in Li-ESWT group were administered Li-ESWT. Cells in the KH-204/Li-ESWT group received combined treatment with $\mathrm{KH}-204$ and LiESWT. Cells in the sham group were controls.

\section{Li-ESWT administration to the HUVECs}

Li-ESWT was administered to the cultured HUVECs using an extracorporeal shockwave machine (CENOWAVE; HNT MEDICAL Co., Ltd, Seoul, Korea). The shockwave probe was kept in contact with the culture dish containing adherent HUVECs by a water-filled cushion covered with common ultrasound gel. The cells were subjected to 200 impulses of Li-ESWT at an energy flux density of $0.09 \mathrm{~mJ} / \mathrm{mm}^{2}$ with a frequency of $120 / \mathrm{min}$. The detail was performed as previously described $(16,18)$. Twenty minutes of Li-ESWT was performed on the adherent HUVECs only once for each passage.

\section{KH-204 preparation}

The major ingredients in $\mathrm{KH}-204$ include five plants: Cornus officinalis (32\%), Lycium chinense (32\%), Rubus coreanus (16\%), Cuscuta chinensis (16\%), and Schisandra chinensis (4\%) and were purchased from a company that produces oriental herbal medicines (KEMIMEDI, Seoul, 
Korea). The quality of each extract was confirmed using high-performance liquid chromatography as previously described $(19,20)$. The marker compounds of Cornus officinalis, Lycium chinense, Rubus coreanus, Cuscuta chinensi, and Schisandra chinensis were identified as loganin, betain, ellagic acid, hyperoside, and Schizandrin, respectively, by the HPLC analyses. Each herb $(20 \mathrm{~kg})$ was extracted in $200 \mathrm{~L}$ of distilled $30 \%$ ethanol and refluxed at $98 \pm 2{ }^{\circ} \mathrm{C}$ for $3 \mathrm{~h}$. The extract was filtered and the liquid from the filtrates was removed by a rotary evaporator and a spray dryer. The side effects and fabrication of KH-204 appeared in a previous publication (21).

\section{KH-204 treatment in vitro and cell tubule formation assay}

Two hundred microliters of BD growth factor reduced (GFR) Matrigel ${ }^{\mathrm{TM}}$ Basement Membrane Matrix (BD Biosciences, Franklin Lakes, NJ, US) was added to a 24-well plate and incubated at $37^{\circ} \mathrm{C}$ for 30 minutes, then HUVECs $\left(1 \times 10^{5}\right.$ cells/well $)$ were incubated in one plate coated with Matrigel in endothelial growth media (EGM) and on a plate coated with endothelial basal media (EBM) (18). $\mathrm{KH}-204(50 \mu \mathrm{g} / \mathrm{mL})$ was added to the plates for two hours in the KH-204 group and KH-204/Li-ESWT groups (10). The other group received an equal amount of medium as a control. After cell adherence, Li-ESWT was performed on the Li-ESWT group and KH-204/Li-ESWT group cells for $20 \mathrm{~min}$. After 12 hours of treatment, the cells were stained using calcein AM dye $(2 \mu \mathrm{g} / \mathrm{mL})$. The formation of cell tubules was observed with an Axio200 fluorescence microscope (Zeiss, Oberkochen, Germany). Images were traced and skeletonized using Image and the angiogenesis tool. The total number of meshes, nodes, and junctions were quantified for each skeleton.

\section{Experimental animals and study design}

Fifty 5-week-old male Sprague-Dawley rats weighing about 230-280 g were purchased from a Korean company (Orient Bio Co., Seongnam, Korea). All animal experiments in this study were approved by the Institutional Animal Care and Use Committee of the Catholic University of Korea (CUMC-2016-0218-01). After fasting, eight rats were randomly chosen as a control group and were injected intraperitoneally with saline. In the other rats, ED was induced by an intraperitoneal injection of streptozotocin (STZ, Sigma Aldrich Chemical Company, St. Louis, MO, US) at a dose of $60 \mathrm{mg} / \mathrm{kg}$ body weight. Only rats with a fasting blood glucose level of $\geq 300 \mathrm{mg} / \mathrm{dL}$ were selected as diabetic and used for further experiments $(\mathrm{N}=36)$. The detail was performed as previously described (18). The selected rats were randomly divided into four groups of eight rates: DMED, DMED + KH-204, DMED + Li-ESWT, and DMED + KH-204/Li-ESWT. Before treatment, all rats received an intraperitoneal injection of $50 \mathrm{mg} / \mathrm{kg}$ EdU (Invitrogen, Carlsbad, CA, US) to identify penile progenitor cells (22).

\section{KH-204 Treatment and Li-ESWT Administration}

After four weeks, during which DMED was established in the rat, DMED + KH-204 and DMED + KH-204/LiESWT rats were fed daily with $400 \mathrm{mg} / \mathrm{kg}$ of $\mathrm{KH}-204$ for one month as previously described (10). Distilled water was fed to the other rats as controls. Meanwhile, rats in the DMED + Li-ESWT and DMED + KH-204/Li-ESWT groups underwent Li-ESWT treatment. In brief, under anesthesia, each rat was placed in the supine position and shaved and the prepuce was degloved. Ultrasound gel was applied to the penis and a shockwave applicator was placed at the penis. A total of 300 shocks were delivered at an energy level of $0.1 \mathrm{~mJ} / \mathrm{mm}^{2}$ and a frequency of 120 shocks/min. This procedure was performed three times a week with one day break for four weeks, which is similar to the treatment course used for patients with ED in clinical practice (6).

\section{Evaluation of erectile function}

Following a 1-week washout period for reducing the side effects of drugs, erectile function was assessed by measuring the intracavernous pressure (ICP) and mean arterial pressure (MAP) under anesthesia. A 23-gauge butterfly needle containing $250 \mathrm{U} / \mathrm{mL}$ heparin solution was carefully inserted into the proximal corpus cavernosum and the other end of the PE-50 tube was connected to a Grass model S48 K pressure transducer (Astro-Med Inc., West Warwick, RI, US) to measure the ICP. A bipolar stainlesssteel electrical stimulator was used to stimulate the major pelvic ganglion with $10 \mathrm{~V}$ for 50 seconds and $2.4 \mathrm{~mA}$ with a pulse width of 2.5 milliseconds. The maximum ICP value of the three stimulations was used for statistical analysis for each rat. The ICP was normalized to the MAP, which was recorded using a BD Intramedic PE-50 tubing (BD, Franklin Lakes, NJ, US) inserted into the left carotid artery at the same time. After erectile function measurements, 
the rats were sacrificed and the penises were harvested for immunohistochemistry and Western blot analyses.

\section{Histology and immunofluorescent staining}

The collected penile samples were fixed in $4 \%$ paraformaldehyde for 24 hours at $4{ }^{\circ} \mathrm{C}$ before embedding in paraffin blocks. The primary antibodies used were as follows: neuronal nitric oxide synthase (nNOS, diluted 1:200; Santa Cruz Biotechnologies, Santa Cruz, CA, US), $\beta$-tubulin (diluted 1:200; Abcam, Cambridge, UK), anti phospho-histone H3 (H3P; diluted 1:200; Santa Cruz Biotechnologies, Santa Cruz, CA, US), isolectin B4 (IB4, diluted 1:500; Cell Signaling Technology, Danvers, MA, US), and caspase-3 (diluted 1:200; Abcam, Cambridge, UK). 6-diamidino-2-phenylindole (DAPI; Vector Laboratories, Inc., Burlingame, CA, US) was used to stain the nuclei. Digital images were obtained using a Zeiss LSM 800 Meta confocal microscope (Zeiss, Oberkochen, Germany) and the mean intensity was calculated using ZEN 2012 (Zeiss, Oberkochen, Germany).

\section{Terminal deoxynucleotidyl transferase dUTP nick end labeling (TUNEL) assay}

Penile paraffin sections were stained by TUNEL using the In Situ Cell Death Detection Kit, TMR red (Roche Diagnostics GmbH, Mannheim, Germany) according to the manufacturer's protocols. The apoptotic index was expressed as the number of TUNEL and DAPI-positive cells in three randomly-chosen sections of the corpus cavernosum per animal, obtained as digital images using a Zeiss LSM 800 Meta confocal microscope (Zeiss, Oberkochen, Germany) and colocalization was calculated using ZEN 2012 (Zeiss, Oberkochen, Germany).

\section{Measurement of oxidative stress}

Oxidative stress in the penile tissue was assessed quantitatively by measuring 8-hydroxy-2-deoxyguanosine (8-OHdG) and superoxide dismutase (SOD) levels using species-specific 8-OHdG and SOD immunoassay ELISA kits (R\&D Systems Europe, Abingdon, UK) according to the manufacturer's instructions. The absorbance was read at a wavelength of $450 \mathrm{~nm}$ in a microplate reader (Synergy H1 M; Biotek, Winooski, VT, US). At least three separate plates of cells were assayed for each clone.

\section{Western blot analyses}

The collected corpus cavernosum tissue was homogenized using ice-cold RIPA buffer (Cell Signaling Technology, Danvers, MA, US) containing an ethylene diamine tetra acetic acid-free protease inhibitor cocktail and phosphatase inhibitor cocktail (Roche Diagnostics GmbH, Mannheim, Germany). Particulate mass was removed by centrifugation $(15,000 \mathrm{~g})$ for $15 \mathrm{~min}$ at $4^{\circ} \mathrm{C}$. Supernatants were analyzed by SDS-PAGE. Primary antibodies used included stromal cellderived factor-1 (SDF-1) diluted 1:200, Abcam, Cambridge, UK), platelet endothelial cell adhesion molecule (PECAM-1) diluted 1:500 (Abcam, Cambridge, UK), and $\beta$-actin diluted 1:1,000 (Abcam, Cambridge, UK).

\section{Statistical analyses}

All data are presented as the mean \pm standard deviation (SD) and were analyzed by SPSS version 22.0 software (IBM, Armonk, NY). One-way ANOVA and Scheffe's test, as appropriate, were used to evaluate whether differences among the groups were significant. A $\mathrm{P}<0.05$ was considered statistically significant.

\section{Results}

\section{KH-204/Li-ESWT significantly improved ED}

Representative images of ICP results are shown in Figure $1 \mathrm{~A}$. The results showed that the ICP of the DMED + KH-204/ Li-ESWT group was higher than that in the DMED group. The quantitative results (Figure 1B) showed that KH-204 combined with Li-ESWT improved DMED. The ratio of ICP/MAP was significantly higher in the DMED + KH-204/ Li-ESWT group than in the DMED + KH-204 $(\mathrm{P}<0.05)$, DMED + Li-ESWT $(\mathrm{P}<0.05)$ and DMED groups $(\mathrm{P}<0.01)$.

\section{Li-ESWT/KH-204 promoted tube formation in vitro and stimulated proliferation in vivo}

To explore cell proliferation after KH-204 and Li-ESWT treatment, we measured the expressions of $\mathrm{H} 3 \mathrm{P}$ and IB4 in the corpus cavernosum. Figure $2 A$ shows that after KH-204 and Li-ESWT treatment, the expression of H3P and IB4 increased, meaning that the administration of $\mathrm{KH}-204$ and Li-ESWT stimulated proliferation in the corpus cavernosum and more angiogenesis. Figure $2 B$ shows the formation of tubes in vitro for each group. Figure $2 C$ shows the quantitative 

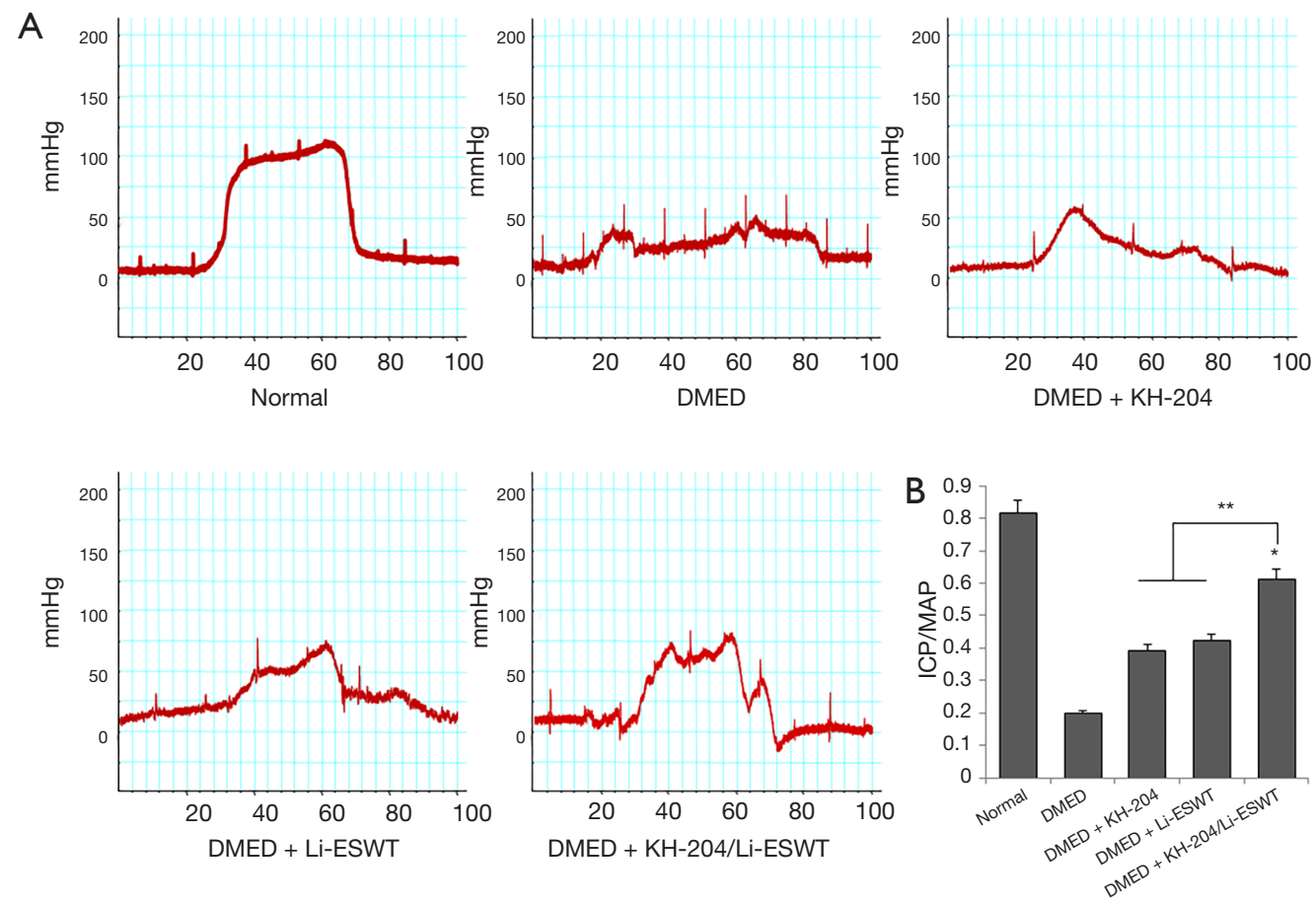

Figure 1 Erectile function of each group. (A) Representative images of intracavernous pressure (ICP) in response to electrical stimulation of the cavernosal nerve; (B) results of the ratio of ICP to the mean MAP of each group. Each bar shows the mean values (standard deviation). *, $\mathrm{P}<0.01$ compared with DMED rats; **, $\mathrm{P}<0.05$ compared with $\mathrm{DM}+\mathrm{ESWT}$ and DM + MSCT groups.

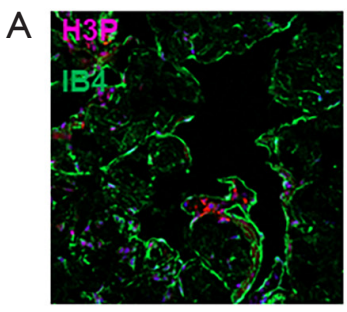

Normal

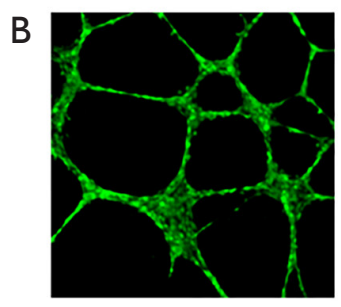

Control

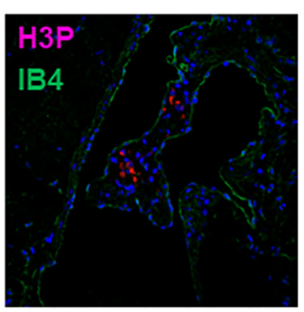

DMED

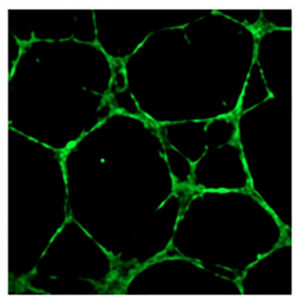

KH-204

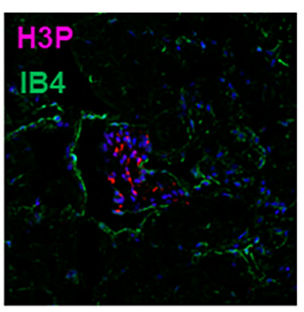

DMED + KH-204

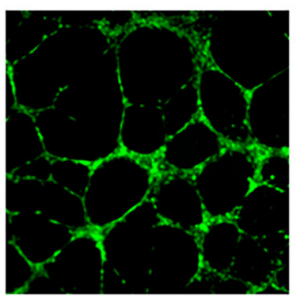

Li-ESWT

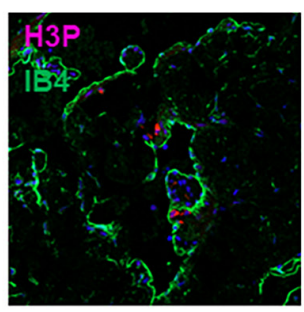

DMED + Li-ESWT

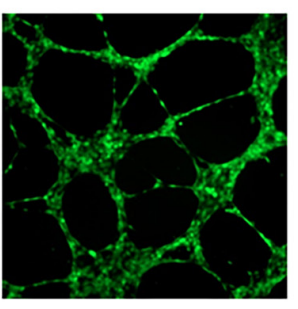

KH-204/Li-ESWT

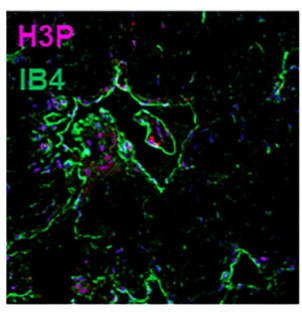

DMED + KH-204/Li-ESWT

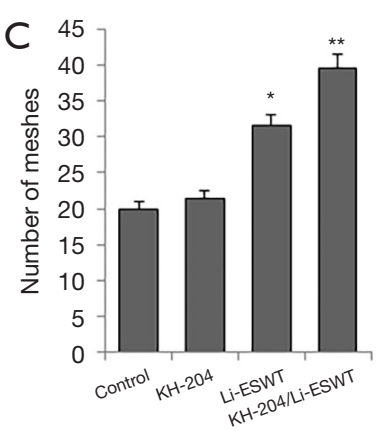

Figure 2 KH-204/Li-ESWT promotes tube formation in vitro and stimulated proliferation in vivo. (A) Representative images of IB4 and H3P. After KH-204 and Li-ESWT treatment, the expression of H3P and IB4 was increased. Original magnification: $\times 200$. (B) Fluorescence microscopy images showing formation of tube network. Original magnification: $\times 50$. (C) Quantitative results of the total meshes in each group. *, $\mathrm{P}<0.05$ compared with DMED group; **, $\mathrm{P}<0.01$ compared with DMED group. 

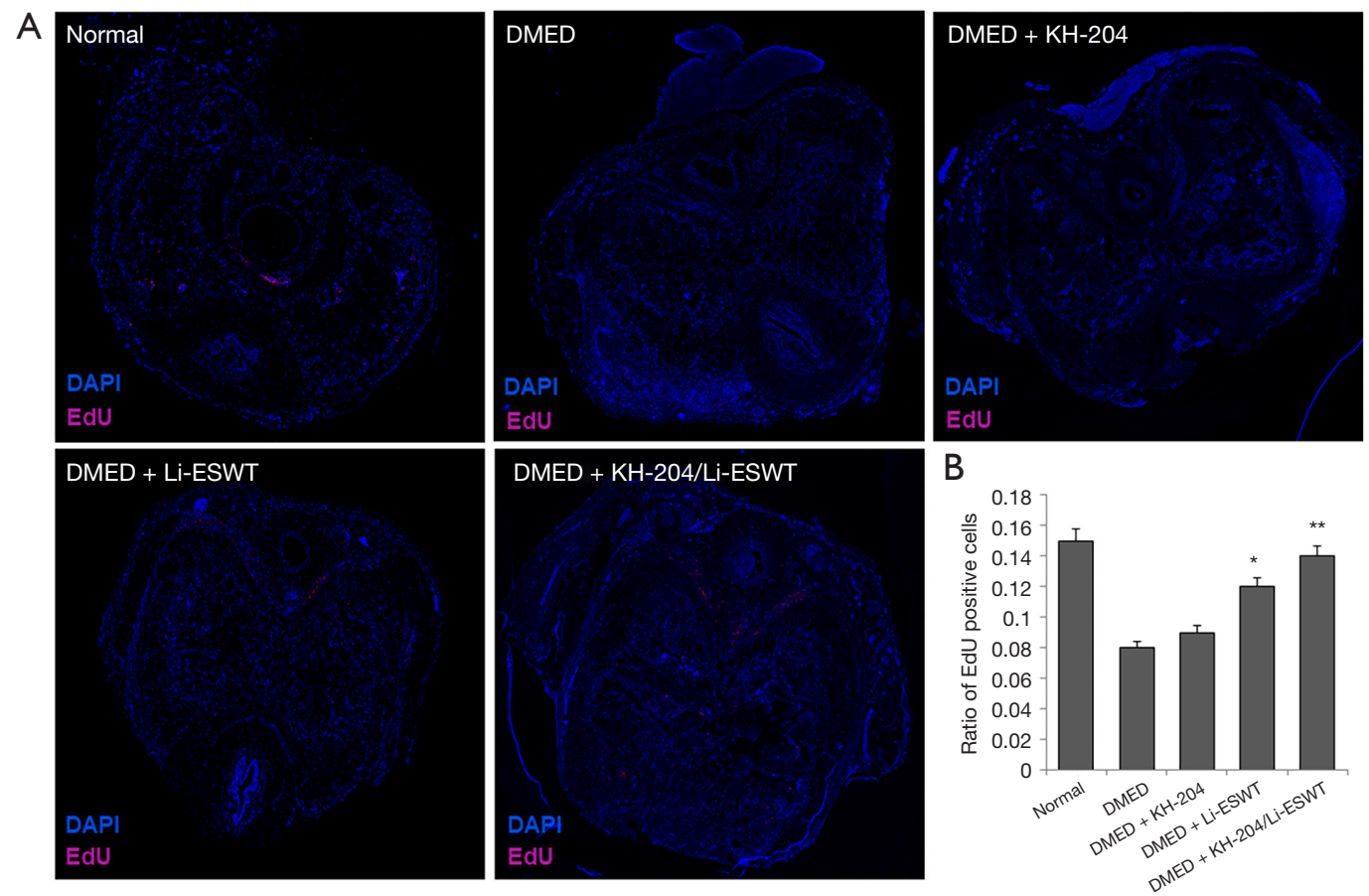

Figure 3 KH-204 maintains penile progenitor cells survival after Li-ESWT homed them to the corpus cavernosum. (A) Representative images of corpus cavernosum. Original magnification: $\times 200$. (B) Quantitative results of EdU-labelled cells. *, $\mathrm{P}<0.05$ compared with DMED group; **, $\mathrm{P}<0.01$ compared with DMED group.

analysis of tube formation and demonstrates that more HUVECs were formed into tubes $(\mathrm{P}<0.01)$ after $\mathrm{KH}-204$ and Li-ESWT treatment compared to the sham group.

\section{KH-204 increased penile progenitor cells survival after Li- ESWT homed them to the corpus cavernosum}

Before treatment, all rats received intraperitoneal injections of $50 \mathrm{mg} / \mathrm{kg} \mathrm{EdU}$. At the fourth week post-injection, we performed immunofluorescence staining to examine the penile progenitor cells in the corpus cavernosum. Figure $3 \mathrm{~A}$ shows the outcome of EdU-labelled penile progenitor cells. Figure $3 B$ shows the quantitative results in each group. After treatment with the combination of $\mathrm{KH}-204$ and Li-ESWT, there were more penile progenitor cells in the corpus cavernosum $(\mathrm{P}<0.01)$ compared to the DMED group and in Li-ESWT-only group, the number of penile progenitor cells was more than in the DMED group $(\mathrm{P}<0.05)$.

\section{Oxidative stress was relieved by $\mathrm{KH}-204 / \mathrm{Li}-\mathrm{ESWT}$ through the Nrf2/HO-1 patbway}

Oxidative stress in the penile tissue was assessed quantitatively by measuring $8-\mathrm{OHdG}$ and SOD levels by ELISA for each group. Figure $4 A$ and $B$ show the results of the 8-OHdG and SOD ELISA analyses. After KH-204 or Li-ESWT, the oxidative stress in the corpus cavernosum was decreased $(\mathrm{P}<0.05)$. However, the combination of KH-204 and Li-ESWT was not different than the single treatments alone. Figure $4 C$ shows the Nrf2 and HO-1 expression in each group after treatment. Figure $4 D$ shows the relative band density of the Western blot results. The Nrf2 and HO-1 expressions after KH-204 or Li-ESWT treatments were higher than in the DMED group $(\mathrm{P}<0.05)$, and the Nrf2 and HO-1 expressions after the combined treatment with KH-204 and Li-ESWT were higher than in the DMED group $(\mathrm{P}<0.01)$. Apparently, the combination of KH-204 and Li-ESWT stimulated the Nrf2/HO-1 pathway to regulate the oxidative stress.

\section{KH-204/Li-ESWT decreased apoptosis in DMED rats}

Apoptosis in the corpus cavernosum was examined by TUNEL. Figure $5 A$ shows the apoptotic cells in the corpus cavernosum for each group. Clearly, after Li-ESWT apoptosis was decreased. Figure $5 B$ presents the apoptotic 
A

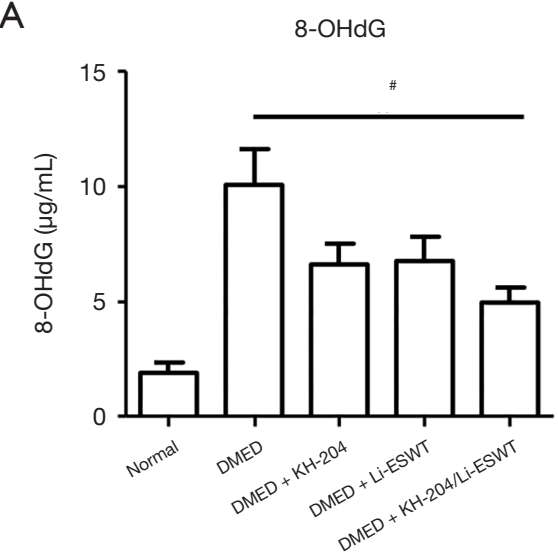

C

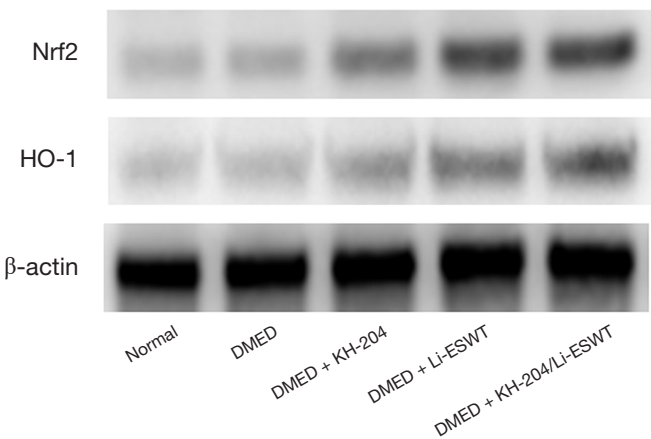

B
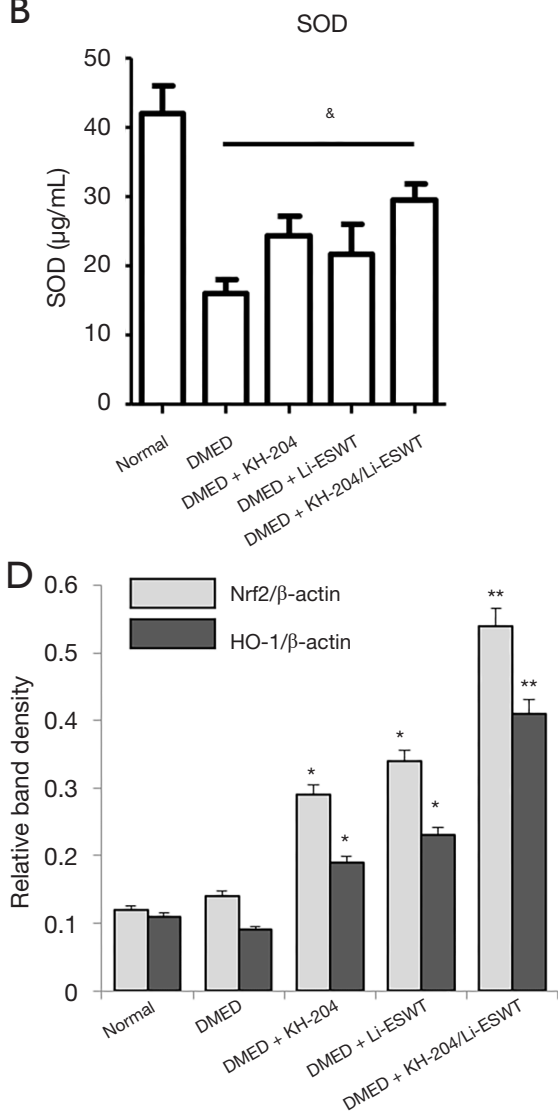

Figure 4 Oxidative stress is relieved by KH-204/Li-ESWT through the Nrf2/HO-1 pathway. (A) and (B) are representative results of 8-OHdG and SOD analyzed by ELISA in each group. " , ${ }^{*}, \mathrm{P}<0.05$ compared to DMED group. (C) Western blot results of each group in the corpus cavernosum. (D) Relative band densities of Western blot results in each group. *, $\mathrm{P}<0.05$ compared to DMED group; **, $\mathrm{P}<0.01$ compared to DMED group.

index for each group. After KH-204 treatment, apoptosis was less than in the DMED group $(\mathrm{P}<0.05)$. And, not only Li-ESWT but the combination of KH-204 and LiESWT, further reduced apoptosis in the corpus cavernosum $(\mathrm{P}<0.01)$.

\section{Li-ESWT increased $n N O S$ expression in the corpus cavernosum}

The nNOS expression in Li-ESWT rats was higher than in those who did not receive Li-ESWT treatment. Figure $6 A$ shows the nNOS expression in each group and Figure $6 B$ shows the quantitative results in each group. After $\mathrm{Li}-\mathrm{ESWT}$, the nNOS expression was higher than in other groups $(\mathrm{P}<0.01)$. However, after $\mathrm{KH}-204$ treatment alone there was no nNOS in the corpus cavernosum.

\section{KH-204/Li-ESWT promoted SDF-1 and PECAM-1 in vivo}

Lastly, the chemotactic factors were explored in vivo for each group. Interestingly, Figure $7 A$ shows the SDF-1 and PECAM-1 expressions were higher after Li-ESWT. Figure $7 B$ shows the quantitative results of Western blots. After Li-ESWT, the expressions of SDF-1 and PECAM-1 were increased in vivo $(\mathrm{P}<0.05)$. The combination of $\mathrm{KH}-$ 204 and Li-ESWT increased SDF-1 and PECAM-1 expressions more $(\mathrm{P}<0.01)$. Perhaps, the KH-204-decreased 


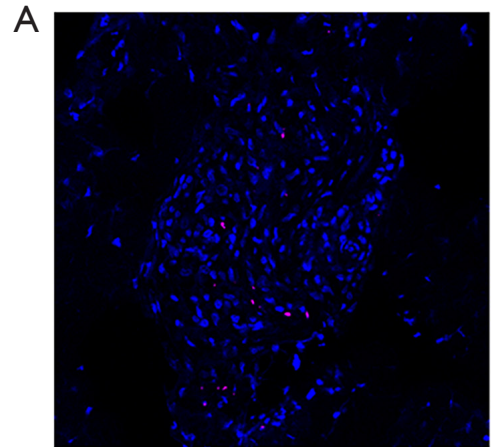

Normal

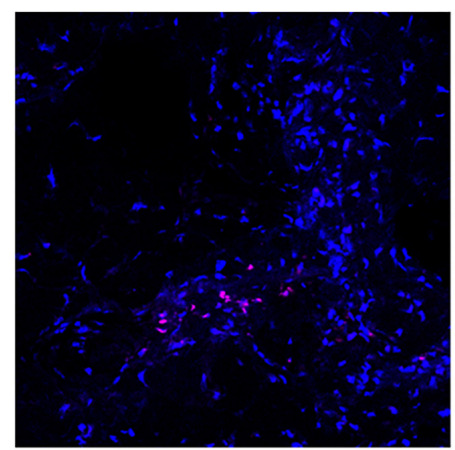

DMED + Li-ESWT

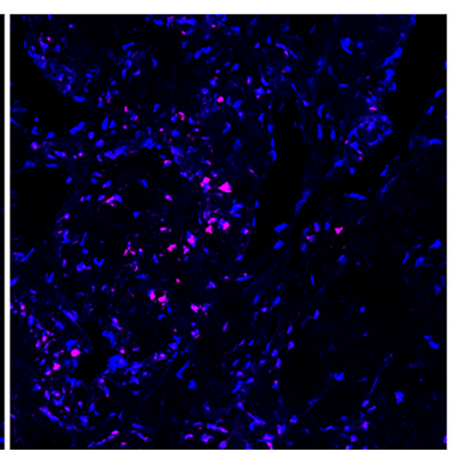

DMED

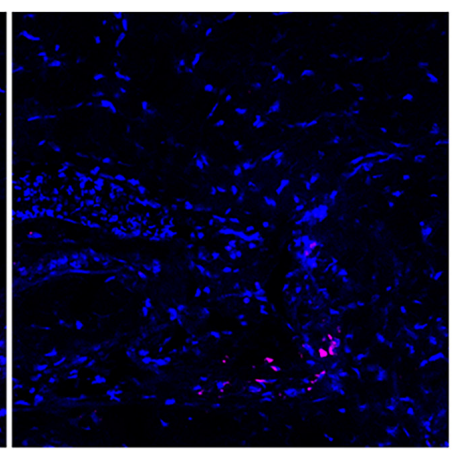

DMED + KH-204/Li-ESWT

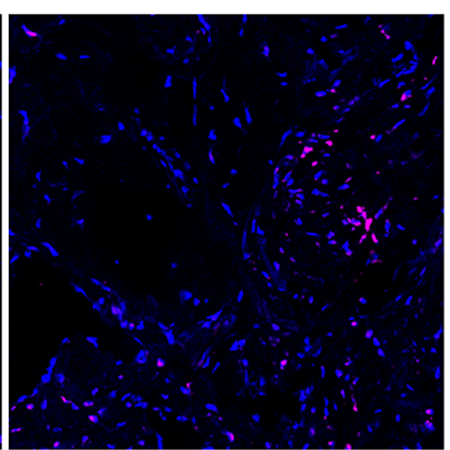

DMED + KH-204

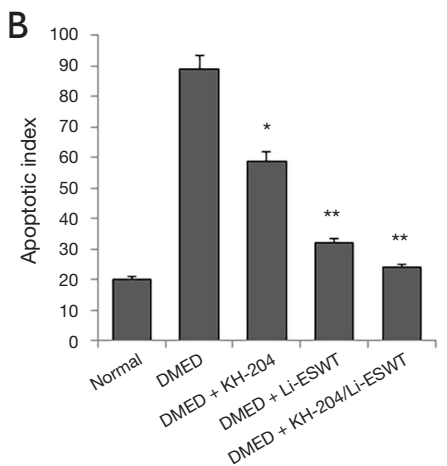

Figure 5 KH-204/Li-ESWT decreases apoptosis in DMED rats. (A) Representative images of TUNEL in the corpus cavernosum. Apoptotic cells are red, blue are DAPI-labelled cells. Original magnification: $\times 200$. (B) Apoptosis index. * $\mathrm{P}<0.05$ compared to DMED group; ${ }^{* *}, \mathrm{P}<0.01$ compared to DMED group.

oxidative stress prevented more penile progenitor cells from moving to the corpus cavernosum, which increased expression of the chemotactic factors.

\section{Discussion}

The risk factors identified for ED include older age, coexisting illnesses, longer duration of diabetes, and poor diabetes control $(23,24)$. Current therapies have been associated with unsatisfactory outcomes (8). Therefore, in this study, we explored a combination treatment, both of which have shown potential for treating DMED and investigated their synergistic effect. Our results confirmed that the combination of Li-ESWT and KH-204 could be an effective approach for DMED by promoting proliferation, homing penile progenitor cells to the corpus cavernosum, and acting as antioxidants.

Stem cells are recognized as a novel and promising treatment because of their ability to self-regenerate and differentiate into various specialized cells (25), including endothelial cells, cavernous smooth muscle cells, and neurons. Only a few studies have investigated the effect of various stem cell types, including bone marrow mesenchymal stem cells, and adipose tissue-derived stem cells, on ED $(26,27)$. However, to explore the effect of the stem cells more locally, Lin et al. applied Li-ESWT to penile progenitor cells (22). In that study, Li-ESWT was found to activate penile progenitor cell in situ. But this research was performed in physiology. As for pathology, especially for DMED, the microenvironment around penile progenitor cells lacks a blood supply. And oxidative stress induced by high glucose in DM also threatens penile progenitor cells $(28,29)$. In this study, our results revealed that in the DMED microenvironment, Li-ESWT could promote angiogenesis and proliferation by activating penile progenitor cells (22), which was consistent with results in physiological studies. Furthermore, after Li-ESWT treatment, there were more penile progenitor cells in the corpus cavernosum. In a previous study, Qiu et al. found that Li-ESWT enhanced recruitment of mesenchymal stem cells (MSCs) in diabetic rats (16). In another study in a bone defect rat model, researchers also found that 

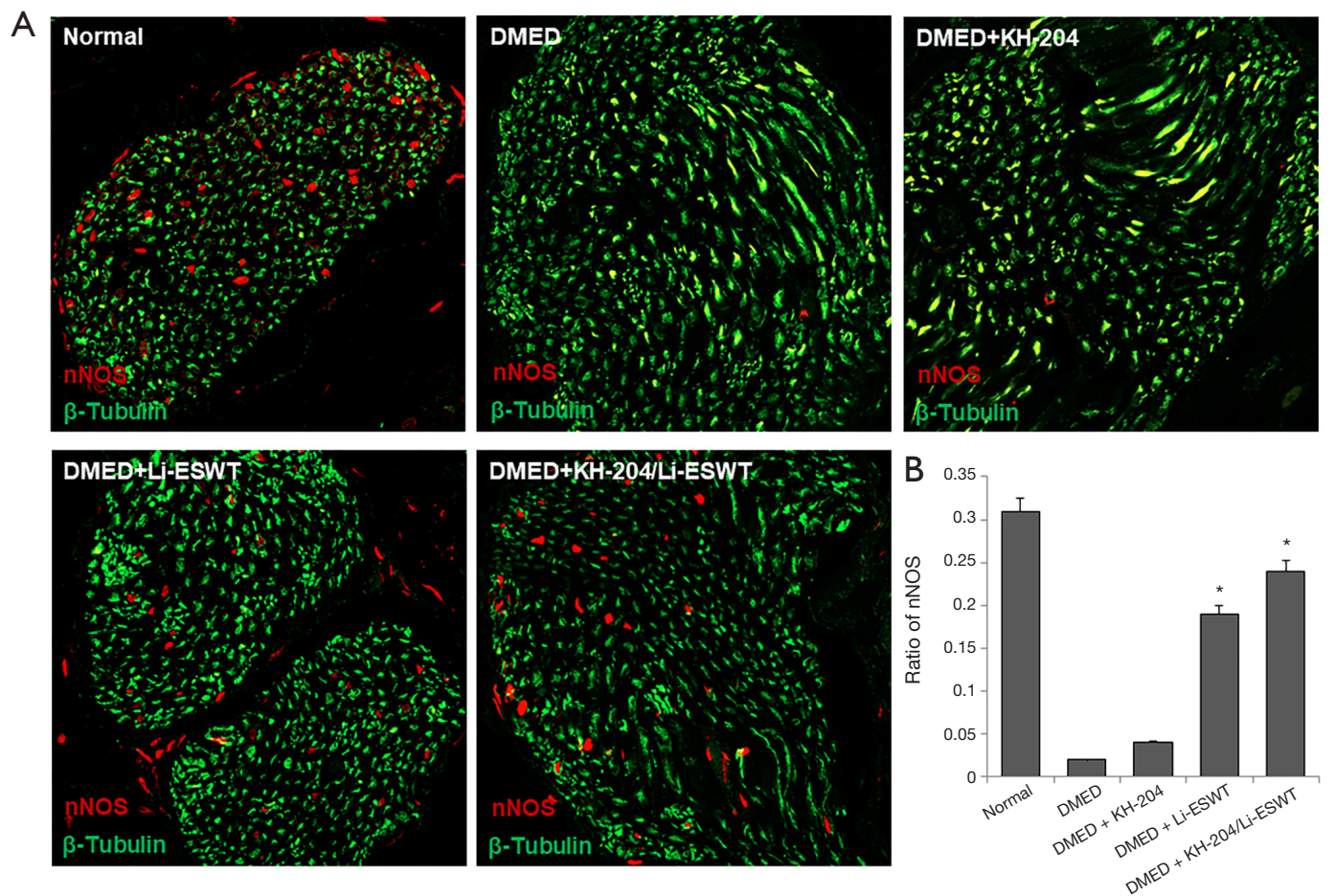

Figure 6 Li-ESWT increases nNOS expression in the corpus cavernosum. (A) Representative images of nNOS staining in the corpus cavernosum; (B) the ratio of positive nNOS in each group. Each bar shows the mean values (standard deviation). Original magnification: $\times 400 .{ }^{*}, \mathrm{P}<0.01$ compared with DMED control group.

A

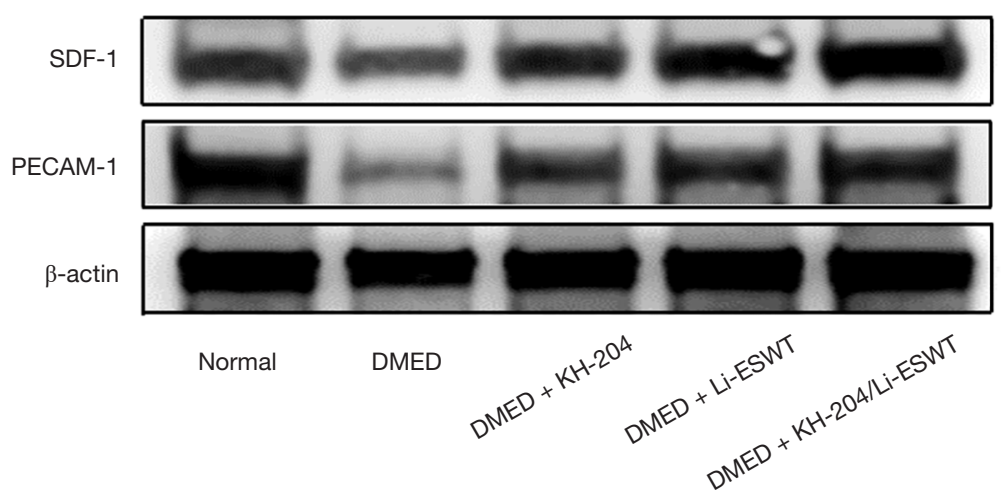

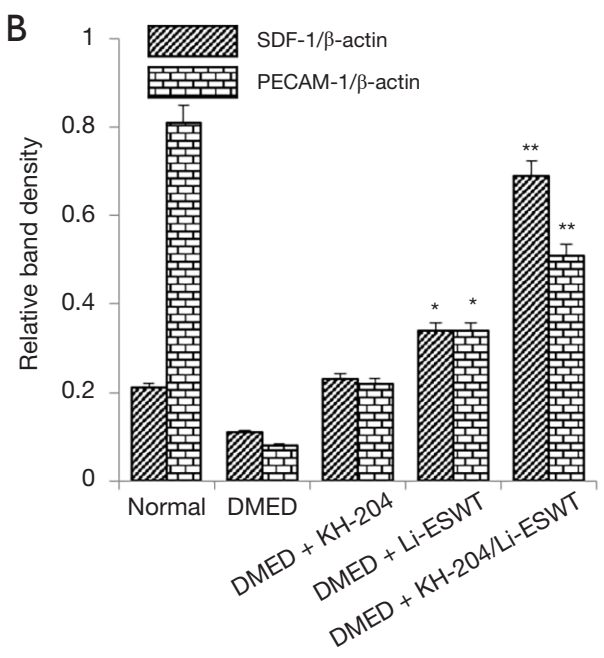

Figure 7 KH-204/Li-ESWT promotes SDF-1 and PECAM-1 expression in vivo. (A) Western blot results of each group in the corpus cavernosum; (B) relative band densitities of the Western blot results in each group. *, $\mathrm{P}<0.05$ compared to DMED group; **, $\mathrm{P}<0.01$ compared to DMED group. 
Li-ESWT resulted in the homing of stem cells (30). In this experiment, chemotactic factors, such as SDF-1 and PECAM, were upregulated after Li-ESWT. The higher expressions of SDF-1 (31) and PECAM drove stem cells to the corpus cavernosum, then transformed to penile progenitor cells.

Stem cells decreased oxidative stress response which contributed to apoptosis (32). And in DMED, reactive oxygen species (ROS) produced by oxidative stress were toxic to stem cells in the corpus cavernosum (33). Even though Li-ESWT recruited progenitor cells, oxidative stress could result in unsatisfactory therapeutic results. So we added KH-204 to our experiment. Meanwhile, Nrf2 and HO-1 as biomarkers of antioxidant were researched in recent studies. Kobayashi et al. found that Nrf2 was a crucial transcription factor which regulates the expression of defensive antioxidants and detoxification enzymes in vitro (34). And Hong et al. illuminated HO-1, as a downstream factor of $\mathrm{Nrf2}$, was also involved in the antioxidant effects (35). In this study, our results showed $\mathrm{KH}-204$ encouraged antioxidation by stimulating the Nfr2/HO-1 pathway. After KH-204 administration, the expression of Nfr2 and HO-1 were increased. Meanwhile, the results illuminated apoptosis in the corpus cavernosum was inhibited by KH-204. So we concluded KH-204 protected progenitor cells from oxidative stress through stimulating Nfr2/HO-1 pathway.

Effective drugs even don't work if they can't reach the target adequately. In this study, we proved that $\mathrm{KH}-$ 204 improved DMED by decreasing oxidative stress in the penile tissue. But, as an oral herbal medicine, $\mathrm{KH}$ 204 must be transmitted to the target tissue in a timely fashion and in adequate amounts, and these requirements cannot be ignored. Due to the lack of blood supply in the corpus cavernosum of DEMD patients, KH-204 cannot satisfactorily arrive at the corpus cavernosum, which means that the delivery KH-204 into target tissues presents a new challenge. To approach this problem, we focused on the advantage of Li-ESWT. In our previous research (36), LiESWT improved angiogenesis in the corpus cavernosum, which meant $\mathrm{KH}-204$ might be able to get into the corpus cavernosum through the hemodynamics enhanced by LiESWT. The results showed that KH-204 combined with LiESWT provided a better DMED treatment and that there was more $\mathrm{KH}-204$ in the corpus cavernosum. However, the concentration of $\mathrm{KH}-204$ in corpus cavernosum could not be tested because KH-204 is a compound of several herbs, which was a shortcoming of this study. Additionally, in this study, the combined therapy was administered at the early stage of DMED, which meant the effect of KH-204 and LiESWT at the middle and advanced stages of DMED were not assessed.

\section{Conclusions}

KH-204 protected penile progenitor cells, which were recruited to the corpus cavernosum by Li-ESWT, from apoptosis via its antioxidative effects. The combination of Li-ESWT and KH-204 could be a potential and efficient synergistic treatment for DMED.

\section{Acknowledgments}

Funding: This research was supported by the Bio and Medical Technology Development Program of the National Research Foundation (NRF) funded by the Ministry of Science and ICT (NRF-2018M3A9E8020861). This research was supported by a grant of the Korea Health Technology R\&D Project through the Korea Health Industry Development Institute (KHIDI), funded by the Ministry of Health \& Welfare, Republic of Korea (grant number : HI19C0310).

\section{Footnote}

Conflicts of Interest: All authors have completed the ICMJE uniform disclosure form (available at http://dx.doi. org/10.21037/tau.2020.01.23). The authors have no conflicts of interest to declare.

Ethical Statement: The authors are accountable for all aspects of the work in ensuring that questions related to the accuracy or integrity of any part of the work are appropriately investigated and resolved. All animal experiments in this study were approved by the Institutional Animal Care and Use Committee of the Catholic University of Korea (CUMC-2016-0218-01).

Open Access Statement: This is an Open Access article distributed in accordance with the Creative Commons Attribution-NonCommercial-NoDerivs 4.0 International License (CC BY-NC-ND 4.0), which permits the noncommercial replication and distribution of the article with the strict proviso that no changes or edits are made and the original work is properly cited (including links to both the formal publication through the relevant DOI and the 
license). See: https://creativecommons.org/licenses/by-nc$\mathrm{nd} / 4.0 /$.

\section{References}

1. Johannes $\mathrm{CB}$, Araujo AB, Feldman HA, et al. Incidence of erectile dysfunction in men 40 to 69 years old: longitudinal results from the Massachusetts male aging study. J Urol 2000;163:460-3.

2. Nunes KP, de Oliveira AA, Szasz T, et al. Blockade of Toll-Like Receptor 4 Attenuates Erectile Dysfunction in Diabetic Rats. J Sex Med 2018;15:1235-45.

3. Isidro ML. Sexual dysfunction in men with type 2 diabetes. Postgrad Med J 2012;88:152-9.

4. Vardi Y, Appel B, Kilchevsky A, et al. Does low intensity extracorporeal shock wave therapy have a physiological effect on erectile function? Short-term results of a randomized, double-blind, sham controlled study. J Urol 2012;187:1769-75.

5. Borrelli F, Colalto C, Delfino DV, et al. Herbal Dietary Supplements for Erectile Dysfunction: A Systematic Review and Meta-Analysis. Drugs 2018;78:643-73.

6. Vardi Y, Appel B, Jacob G, et al. Can low-intensity extracorporeal shockwave therapy improve erectile function? A 6-month follow-up pilot study in patients with organic erectile dysfunction. Eur Urol 2010;58:243-8.

7. Zhu GQ, Jeon SH, Bae WJ, et al. Efficient Promotion of Autophagy and Angiogenesis Using Mesenchymal Stem Cell Therapy Enhanced by the Low-Energy Shock Waves in the Treatment of Erectile Dysfunction. Stem Cells Int 2018;2018:1302672.

8. Martínez-Jabaloyas JM, Gil-Salom M, Villamon-Fort $\mathrm{R}$, et al. Prognostic factors for response to sildenafil in patients with erectile dysfunction. Eur Urol 2001;40:6416; discussion 647.

9. Park CS, Ryu SD, Hwang SY. Elevation of intracavernous pressure and NO-cGMP activity by a new herbal formula in penile tissues of aged and diabetic rats. J Ethnopharmacol 2004;94:85-92.

10. Bae WJ, Zhu GQ, Choi SW, et al. Antioxidant and Antifibrotic Effect of a Herbal Formulation In Vitro and in the Experimental Andropause via Nrf2/HO-1 Signaling Pathway. Oxid Med Cell Longev 2017;2017:6024839.

11. Castela Â, Costa C. Molecular mechanisms associated with diabetic endothelial-erectile dysfunction. Nat Rev Urol 2016;13:266-74.

12. Cayton T, Harwood AE, Smith GE, et al. Extracorporeal shockwave therapy for the treatment of lower limb intermittent claudication: study protocol for a randomised controlled trial (the SHOCKWAVE 1 trial). Trials 2017;18:104.

13. Becker M, Goetzenich A, Roehl AB, et al. Myocardial effects of local shock wave therapy in a Langendorff model. Ultrasonics 2014;54:131-6.

14. Young SR, Dyson M. The effect of therapeutic ultrasound on angiogenesis. Ultrasound Med Biol 1990;16:261-9.

15. Behr-Roussel D, Giuliano F. Low-energy shock wave therapy ameliorates erectile dysfunction in a pelvic neurovascular injuries rat model. Transl Androl Urol 2016;5:977-9.

16. Qiu X, Lin G, Xin Z, et al. Effects of low-energy shockwave therapy on the erectile function and tissue of a diabetic rat model. J Sex Med 2013;10:738-46.

17. Alhazzani A, Rajagopalan P, Albarqi Z, et al. Mesenchymal Stem Cells (MSCs) Coculture Protects [Ca2+]i Orchestrated Oxidant Mediated Damage in Differentiated Neurons In Vitro. Cells 2018. doi: 10.3390/cells7120250.

18. Jeon SH, Zhu GQ, Bae WJ, et al. Engineered Mesenchymal Stem Cells Expressing Stromal Cell-derived Factor-1 Improve Erectile Dysfunction in StreptozotocinInduced Diabetic Rats. Int J Mol Sci 2018. doi: 10.3390/ ijms19123730.

19. Choi SW, Jeon SH, Kwon EB, et al. Effect of Korean Herbal Formula (Modified Ojayeonjonghwan) on Androgen Receptor Expression in an Aging Rat Model of Late Onset Hypogonadism. World J Mens Health 2019;37:105-12.

20. Bae WJ, Ha US, Choi JB, et al. Protective Effects of KH204 in the Bladder of Androgen-Deprived Rats. World J Mens Health 2015;33:73-80.

21. Kim SJ, Kim MR, Hwang SY, et al. Preliminary report on the safety of a new herbal formula and its effect on sperm quality. World J Mens Health 2013;31:254-61.

22. Lin G, Reed-Maldonado AB, Wang B, et al. In Situ Activation of Penile Progenitor Cells With LowIntensity Extracorporeal Shockwave Therapy. J Sex Med 2017;14:493-501.

23. Azad AK, Setunge S, Selim S, et al. Dyslipidaemia as a risk factor for erectile dysfunction in type 2 diabetes mellitus patients. Diabetes Metab Syndr 2019;13:748-53.

24. Al Demour S, Jafar H, Adwan S, et al. Safety and Potential Therapeutic Effect of Two Intracavernous Autologous Bone Marrow Derived Mesenchymal Stem Cells injections in Diabetic Patients with Erectile Dysfunction: An Open Label Phase I Clinical Trial. Urol Int 2018;101:358-65. 25. Soebadi MA, Moris L, Castiglione F, et al. Advances 
in stem cell research for the treatment of male sexual dysfunctions. Curr Opin Urol 2016;26:129-39.

26. Ouyang X, Han X, Chen Z, et al. MSC-derived exosomes ameliorate erectile dysfunction by alleviation of corpus cavernosum smooth muscle apoptosis in a rat model of cavernous nerve injury. Stem Cell Res Ther 2018;9:246.

27. Yang J, Zhang Y, Zang G, et al. Adipose-derived stem cells improve erectile function partially through the secretion of IGF-1, bFGF, and VEGF in aged rats. Andrology 2018;6:498-509.

28. Tian W, Lei H, Guan R, et al. Icariside II ameliorates diabetic nephropathy in streptozotocin-induced diabetic rats. Drug Des Devel Ther 2015;9:5147-57.

29. Yan J, Young ME, Cui L, et al. Increased glucose uptake and oxidation in mouse hearts prevent high fatty acid oxidation but cause cardiac dysfunction in diet-induced obesity. Circulation 2009;119:2818-28.

30. Chen YJ, Wurtz T, Wang CJ, et al. Recruitment of mesenchymal stem cells and expression of TGF-beta 1 and VEGF in the early stage of shock wave-promoted bone regeneration of segmental defect in rats. J Orthop Res 2004;22:526-34.

31. Fandel TM, Albersen M, Lin G, et al. Recruitment of intracavernously injected adipose-derived stem cells to

Cite this article as: Jeon SH, Bae WJ, Zhu GQ, Tian W, Kwon EB, Kim GE, Hwang SY, Lee KW, Cho HJ, Ha US, Hong SH, Lee JY, Kim SW. Combined treatment with extracorporeal shockwaves therapy and an herbal formulation for activation of penile progenitor cells and antioxidant activity in diabetic erectile dysfunction. Transl Androl Urol 2020;9(2):416427. doi: $10.21037 /$ tau.2020.01.23 the major pelvic ganglion improves erectile function in a rat model of cavernous nerve injury. Eur Urol 2012;61:201-10.

32. Honda F, Kano H, Kanegane H, et al. The kinase Btk negatively regulates the production of reactive oxygen species and stimulation-induced apoptosis in human neutrophils. Nat Immunol 2012;13:369-78.

33. Liu GY, Jiang XX, Zhu X, et al. ROS activates JNKmediated autophagy to counteract apoptosis in mouse mesenchymal stem cells in vitro. Acta Pharmacol Sin 2015;36:1473-9.

34. Kobayashi A, Ohta T, Yamamoto M. Unique function of the Nrf2-Keap1 pathway in the inducible expression of antioxidant and detoxifying enzymes. Methods Enzymol 2004;378:273-86.

35. Hong B, Su Z, Zhang C, et al. Reserpine Inhibit the JB6 $\mathrm{P}+$ Cell Transformation Through Epigenetic Reactivation of Nrf2-Mediated Anti-oxidative Stress Pathway. AAPS J 2016;18:659-69.

36. Jeong HC, Jeon SH, Qun ZG, et al. Effects of NextGeneration Low-Energy Extracorporeal Shockwave Therapy on Erectile Dysfunction in an Animal Model of Diabetes. World J Mens Health 2017;35:186-95. 